\title{
Towards Resolving the Atomization Process of an Idealized Fire Sprinkler with VOF Modeling
}

\author{
K. V. Meredith*, X. Zhou, and Y. Wang \\ Research Division, FM Global, USA \\ *Corresponding author: karl.meredith@fmglobal.com
}

\begin{abstract}
Fire suppression modeling depends on accurate characterization of the atomization processes of fire sprinklers. Numerical modeling, particularly the volume of fluid (VOF) method, has been applied to understanding the atomization behavior of an idealized sprinkler geometry consisting of a $9.5 \mathrm{~mm}$ inner-diameter cylindrical nozzle and a flat, $25.4 \mathrm{~mm}$ diameter disk with a liquid flow rate of $0.87 \mathrm{~L} / \mathrm{s}$. The simulations have been performed with an OpenFOAM based VOF solver, using the isoAdvector scheme for interfacial reconstruction. The sheet breakup distance and film thickness were calculated and compared with measurements from a previous study. A mesh refinement study identified the sensitivities in the predicted quantities to mesh resolution. This study enables further application of the model to simulation of the fully atomized spray.
\end{abstract}

\section{Keywords}

Fire suppression, primary atomization, VOF, sheet breakup distance, film thickness, sprinkler patternation

\section{Introduction}

The atomization of water by a fire sprinkler is of great interest for fire suppression research. The resulting droplet velocity, diameter, and liquid volume flux largely determine the suppression effectiveness. For example, large droplets can easily penetrate through a fire plume, while small droplets tend to be easily evaporated or carried away with the fire plume and have difficulty reaching the burning surfaces.

Traditionally, atomization in fire sprinklers has been studied experimentally [1]. A great amount of effort has been invested in measuring the sprinkler droplet diameter distribution, droplet velocities, and mass flux profiles [1-7]. These measurements have been used to better understand the atomization process and to develop spray injection models for use within fire suppression modeling [8].

Since the appearance of the first VOF model [9], computational methods associated with VOF have greatly improved in the areas of fidelity and usability [10-13]. VOF simulations provide insight into the underlying processes critical for primary and secondary atomization. With modeling, sprinkler geometry and operating conditions can potentially be varied in a parametric fashion to better understand sprinkler operation. Once the model has been adequately validated, the resulting predictions for droplet diameter, velocity, and liquid volume flux can be directly used in fire suppression CFD simulations as an injection profile derived from first principles.

The goal of this work is to demonstrate the feasibility of using VOF modeling to adequately capture key aspects of sprinkler atomization in an idealized sprinkler geometry, and to provide a path forward to subsequently simulate realistic sprinkler geometries. Accurately resolving the key flow features in the near-field, such as film thickness and sheet breakup length, is critical to enable eventual simulation of the fully atomized spray. Comparisons of the predicted flow features are made with a previously characterized idealized fire sprinkler geometry [14]. A mesh refinement study is performed for better understanding of the required resolution necessary to enable accurate representation of the liquid surface and the details of the subsequent breakup.

\section{Technical Approach}

\section{Experimental Configuration}

The experiment of Zhou and Yu [14] was used for model comparison and validation. The idealized sprinkler used in this experiment consists of a horizontal disk placed beneath a vertical, cylindrical nozzle. Being idealized, there are no slots, tines, frame arms, or boss elements that typically are present in a realistic sprinkler. Three disk diameters were considered: $25.4 \mathrm{~mm}, 38.1 \mathrm{~mm}$, and $50.8 \mathrm{~mm}$. The nozzle was placed $20 \mathrm{~mm}$ above the disk top surface and had an inner diameter of $9.5 \mathrm{~mm}$. 

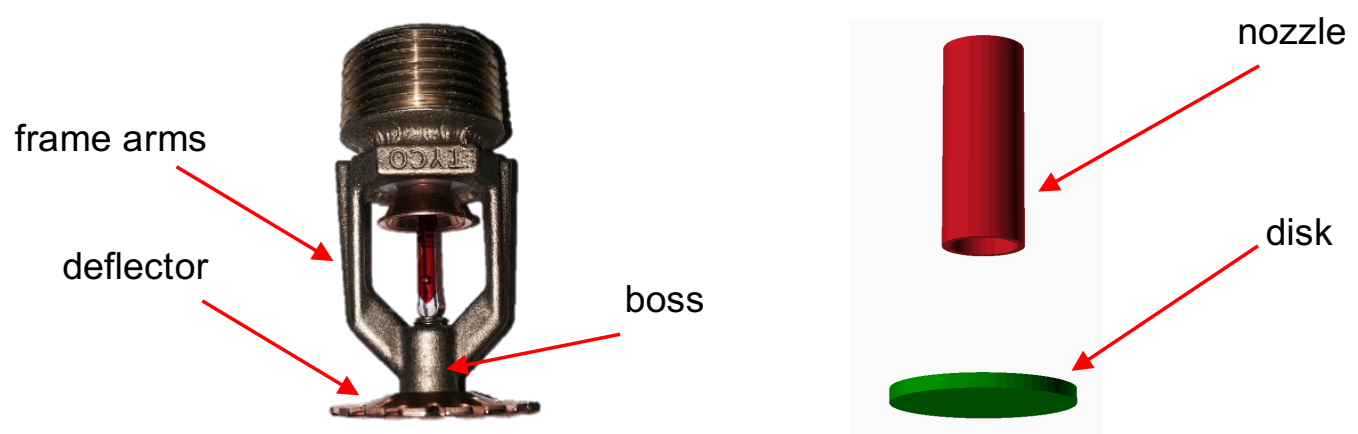

Figure 1. Comparison of a) typical sprinkler geometry showing the frame arms, the boss, and deflector; and b) with idealized sprinkler geometry showing the nozzle and disk.

Water discharge pressures ranging from 0.034 bar to 0.83 bar were used to investigate the spray formation as affected by sprinkler geometry and operating pressure using a laser-based shadow imaging system. The water film thickness (only measured for the lower end of the tested range of discharge pressures), sheet breakup distance, and drop size distributions were measured. An example of the measurements is shown in Figure 2. Refer to Ref. [14] for further details.

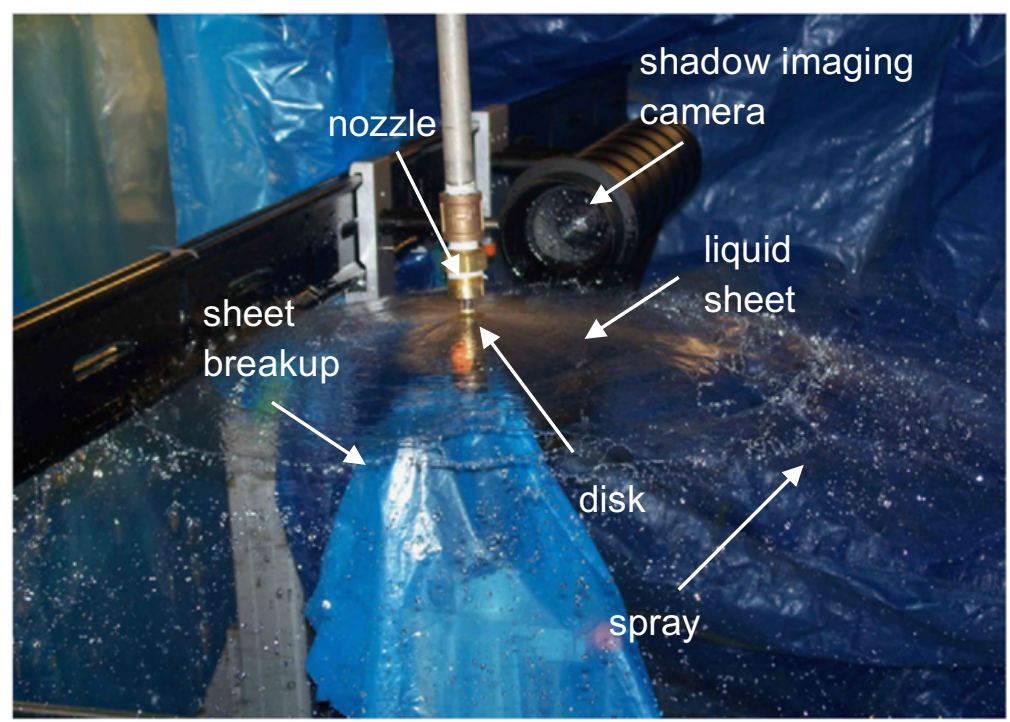

Figure 2. Experimental measurements of atomization process for an idealized fire sprinkler [14].

\section{Numerical Model}

The sprinkler simulations utilized a VOF solver, navalFoam, implemented in foam-extend [15], a community driven fork of the OpenFOAM [16] CFD software. The equations solved in navalFoam have been adequately documented elsewhere [11-13], and portions of the model are reproduced here for reference purposes only. Equations (1-3) represent the continuity, momentum, and phase volume fraction transport equations, respectively,

$$
\boldsymbol{\nabla} \cdot \mathbf{U}=0
$$

$$
\begin{gathered}
\frac{\partial(\rho \mathbf{U})}{\partial t}+\boldsymbol{\nabla} \cdot(\rho \mathbf{U U})-\boldsymbol{\nabla} \cdot\left(\mu_{e f f} \boldsymbol{\nabla} \mathbf{U}\right) \\
=-\boldsymbol{\nabla} p_{d}-\mathbf{g} \cdot \mathbf{x} \boldsymbol{\nabla} \rho+\boldsymbol{\nabla} \mathbf{U} \cdot \boldsymbol{\nabla} \mu_{e f f}+\sigma \kappa \boldsymbol{\nabla} \alpha \\
\frac{\partial \alpha}{\partial t}+\boldsymbol{\nabla} \cdot(\mathbf{U} \alpha)=0
\end{gathered}
$$


where $\mathbf{U}$ represents the velocity vector, $\alpha$ represents the phase fraction, $\rho$ is the density (assumes a combination of phase densities weighted by respective phase fractions), $\mathbf{g}$ is the gravity vector, and $\mu_{e f f}$ is the effective dynamic viscosity from turbulence modeling. The phase fraction, $\alpha$, will only have values between 0 and 1 over the few cells spanning the interface between fluids.

The r.h.s. of Equation 2 represents the pressure body force, the gravity body force, the variation of dynamic viscosity across the interface, and the interfacial surface tension effects. The dynamic pressure is represented as

$$
p_{d}=p-\rho \mathbf{g} \cdot \mathbf{x}
$$

where $\mathbf{x}$ represents the position vector. For the surface tension effect, $\sigma$ represents the liquid surface tension and $\kappa$ represents the mean curvature of the free surface.

$$
\kappa=-\boldsymbol{\nabla} \cdot\left(\frac{\nabla \gamma}{|\nabla \gamma|}\right)
$$

The surface tension force has a finite value only at the interface between phases [17]. Rather than using typical interface compression schemes [18] to try to maintain a sharp interface, navalFoam includes the isoAdvector scheme [11] for approximating a geometric reconstruction of the interface. This scheme explicitly reconstructs a phase interface in each computational cell where $0<\alpha<1$ (i.e., at the intersection between the two phases). This interface is advected through the cell. When calculating fluxes through cell faces, the interface information is used to determine the relative amounts of phase 1 or phase 2 to be advected out of the cell. This approach results in the ability to strongly limit numerical diffusion of the interface. A detailed description of the isoAdvector scheme is beyond the scope of this work, but additional details can be found in Ref. [11].

Large eddy simulation (LES) was used to treat turbulence, and the one-equation eddy model was used for simulating the turbulent kinetic energy. Additional details of the navalFoam model can be found in Refs. [12, 13].

\section{Simulated Geometry}

The simulated geometry, shown in Figure 4, consists of a cylindrical nozzle, $r=4.25 \mathrm{~mm}$ and $l=28 \mathrm{~mm}$, where $r$ is the inner radius and $l$ is the length. The pipe flow inside the nozzle is simulated, having the nozzle inlet at the top of the domain. A disk with diameter $d=12.7 \mathrm{~mm}$ is placed $20 \mathrm{~mm}$ below the nozzle outlet. The disk thickness is set to $2 \mathrm{~mm}$. The overall domain bounds are $x=[-36 \mathrm{~mm}, 288 \mathrm{~mm}], y=[-36 \mathrm{~mm}, 36 \mathrm{~mm}]$, and $z=$ $[-24 \mathrm{~mm}, 48 \mathrm{~mm}]$. The reason for the asymmetry in the $x$-direction is to allow for simulation of the breakup length of the liquid sheet, which for the flow rates studied in Ref. [14], ranges from $153 \mathrm{~mm}$ to $223 \mathrm{~mm}$ for this disk size. Simulating the sheet breakup distance in only one direction allows for a minimization of the required computational cells, and should not affect the flow to any significant extent due to the supercritical nature of the flow (i.e., Froude number $\gg 1$ ). The top, bottom, and sides of the domain are open to the ambient.

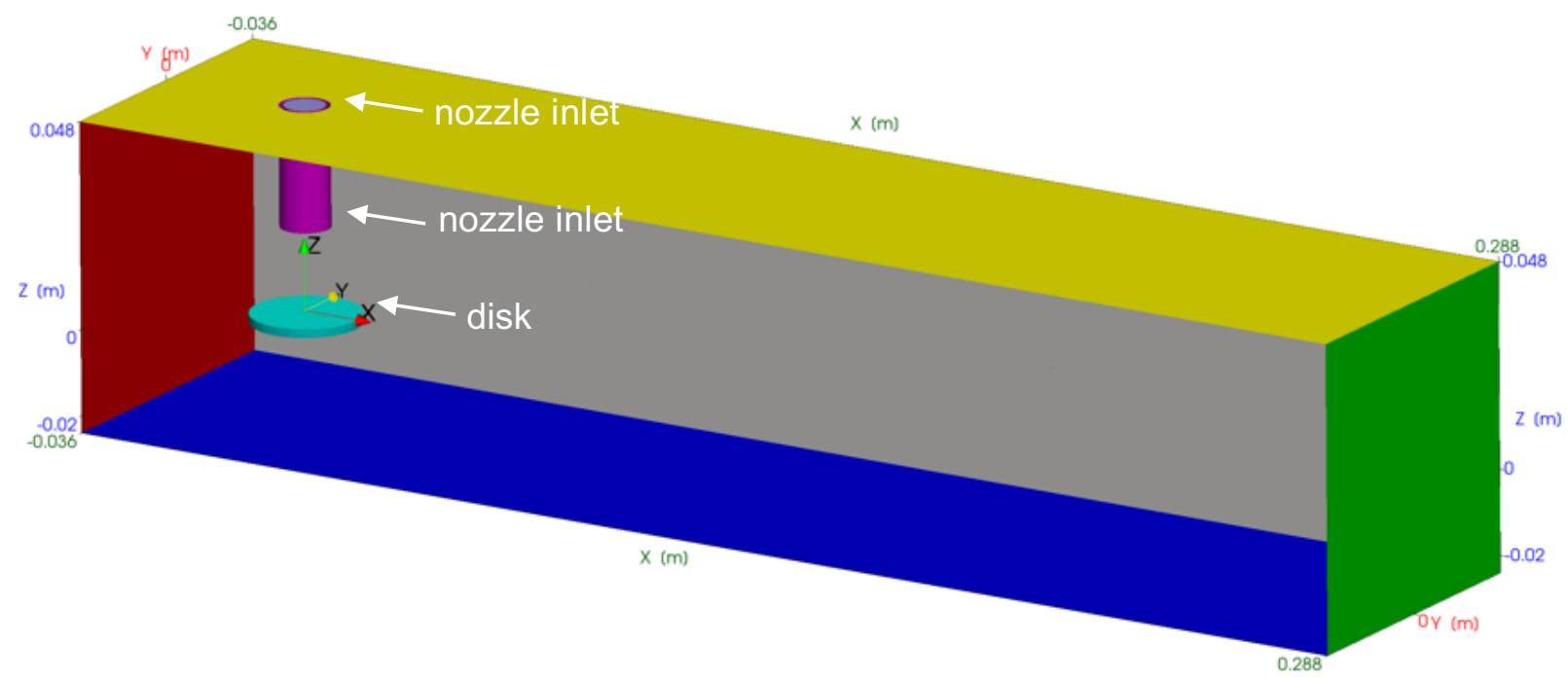

Figure 3. Simulated geometry showing the cylindrical nozzle oriented above the cylindrical disk with inlet, top, side, and bottom boundaries shown. 
The boundary conditions employed in the simulation are listed in Table 1, and correspond to the boundary patches shown in Figure 3. To improve stability in the simulation, the inlet velocity was ramped over time from $U=0 \mathrm{~m} / \mathrm{s}$ at $t=0 \mathrm{sec}$ to $U=12.2 \mathrm{~m} / \mathrm{s}$ at $t=5 \mathrm{~ms}$ and then held constant. This final velocity corresponds to a volumetric flow rate of $52 \mathrm{lpm}$. The inlet was set to a phase fraction of one, and represents the only source of water inflow into the domain. The nozzle and disk wall boundaries consisted of a no-slip velocity condition and a Spallart Allmaras wall function treatment for the subgrid-scale viscosity [19]. The initial conditions were set to quiescent flow and a zero liquid volume fraction. For this study, no turbulent fluctuations were specified at the inlet boundary. This assumption warrants further investigation in future studies, as an operating sprinkler will likely have large fluctuations at the inlet due to pump frequencies, turbulent pipe flow, and flow turning effects from the feed line (typically oriented perpendicular to the nozzle).

Table 1. Simulated boundary conditions.

Inlet

Sides/top/bottom Nozzle/disk

\begin{tabular}{r|l|l|l|} 
Liquid phase fraction & Fixed value $(1)$ & Inlet/outlet & Zero gradient \\
\cline { 2 - 4 } Velocity & Time varying fixed value & No-slip & Pressure inlet/outlet \\
\cline { 2 - 4 } Turbulent kinetic energy & Fixed value $\left(1 \times 10^{-5}\right)$ & Inlet/outlet & Fixed value $(0)$ \\
\cline { 2 - 4 } Pressure & Zero gradient & Total pressure & Zero gradient \\
\cline { 2 - 4 } Subgrid-scale viscosity & Zero gradient & Zero gradient & Spallart Allmaras wall function [19] \\
\cline { 2 - 4 } & &
\end{tabular}

\section{Computational Mesh}

The grid requirements for a VOF simulation to be able to accurately resolve drop sizes is typically on the order of 5 computational cells across the targeted droplet diameter or liquid sheet thickness. For fire sprinkler atomization, the volume mean diameter is approximately $1 \mathrm{~mm}$, with the smallest drop size of interest being on the order of 0.1 $\mathrm{mm}$ diameter. Thus, to resolve these droplets a minimum grid resolution of $\sim 20 \mu \mathrm{m}$ would be necessary. For a uniform grid spacing the number of cells is directly proportional to $\Delta x^{-3}$, and therefore has the potential for resulting in a requirement for a very large number of computational cells. One of the main goals of this research was to identify the minimum grid spacing to resolve the sheet breakup length and most of the breakup processes. Ultimately, adaptive mesh refinement will be required to capture the finest resolutions of interest.

VOF has the potential to predict the liquid volume-flux with a high degree of certainty without necessarily fully resolving each individual droplet. The reason for this is two-fold: 1) VOF accurately conserves the mass of the liquid, and 2) the spatial distribution of liquid volumetric flux is largely dictated by interaction of the solid-core liquid jet emerging from the nozzle with the macroscopic geometry of the idealized sprinkler. Thus, for practicality, minimum mesh resolutions in this study ranged from $1 \mathrm{~mm}$ to $0.25 \mathrm{~mm}$. While these mesh resolutions will certainly not be adequate to capture the finest features of the atomization process (e.g., drop sizes $\approx 0.2 \mathrm{~mm}$ and less), larger features of the flow such as sheet thickness $(\approx 0.8$ to $1.0 \mathrm{~mm})$, ligaments, and the droplets carrying most the liquid volume $\left(D_{v 50} \approx 1 \mathrm{~mm}\right)$ for this condition [14] should be able to be resolved, especially with the finest mesh resolution.

The computational mesh was created by the standard OpenFOAM mesh generation software, snappyHexMesh. The bounds of the simulated domain are shown in Figure 4. One of the goals of this work is to identify the sensitivity of the solution to the mesh refinement. To that end, three mesh sizes were considered. Localized mesh refinement was used to resolve the expected regions of the liquid flow and minimize cell count. The local refinement consisted of three regions: 1) a cylindrical region extending from the bottom of the nozzle to the top of the disk with $r=8 \mathrm{~mm}$, and 2) another cylindrical region with a radius extending beyond the bounds of the computational mesh, with the top and bottom of the cylinder being positioned at $x=-4 \mathrm{~mm}$ and $x=5 \mathrm{~mm}$, respectively. The resolution in these refinement zones was set to values of $\Delta x_{\min }=1 \mathrm{~mm}, \Delta x_{\min }=0.5 \mathrm{~mm}$, and $\Delta x_{\min }=0.25 \mathrm{~mm}$ for the three meshes. The background mesh for each simulation was $\Delta x=4 \mathrm{~mm}$. 
a)

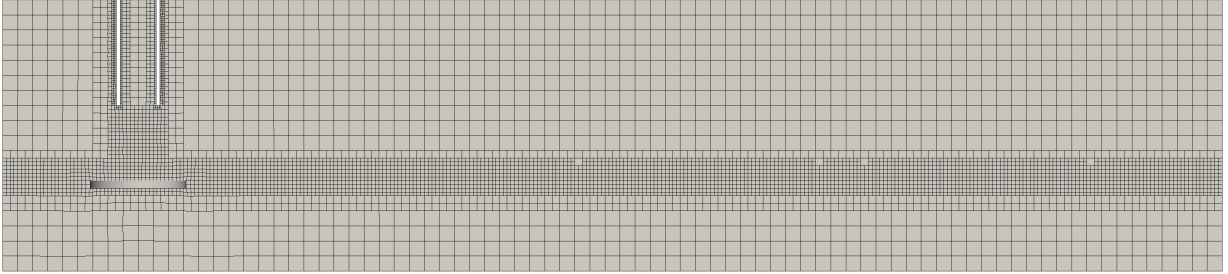

b)

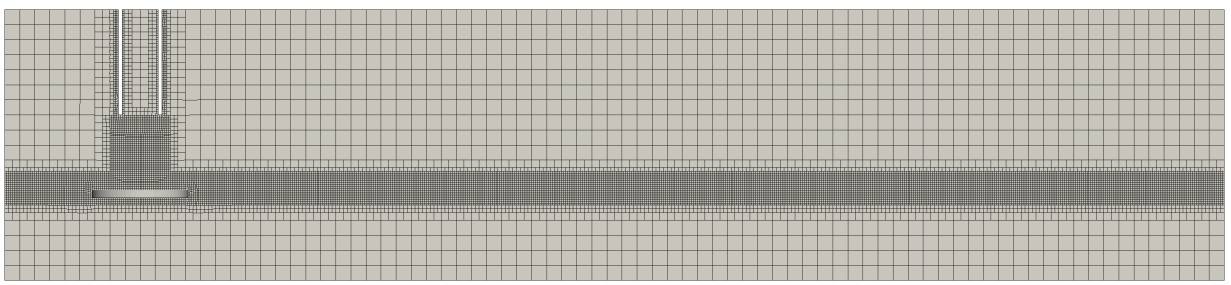

c)

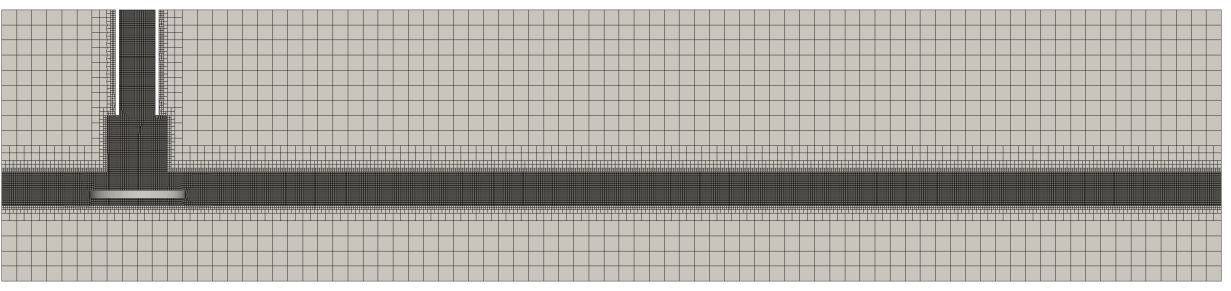

Figure 4. Depictions of the three levels of mesh refinements for the idealized sprinkler geometry: a) $\Delta x_{\min }=1 \mathrm{~mm}$, b) $\Delta x_{\min }=$ $0.5 \mathrm{~mm}$, and c) $\Delta x_{\min }=0.25 \mathrm{~mm}$.

The resulting mesh sizes were $0.3 \mathrm{M}, 1.8 \mathrm{M}$, and $14.1 \mathrm{M}$ respectively. The finest mesh size was also refined within the nozzle region, as this allowed greater stability of the solution while the interface was transiting through this region. The meshes consists of mainly hexahedral cells ( $>98 \%$ of the total cell count) with only a small number of tetrahedral and prismatic cells needed to accurately represent the curvature of the nozzle and disk.

\section{Results}

Each mesh resolution was simulated for a flow rate of $0.87 \mathrm{~L} / \mathrm{s}$ entering the nozzle inlet. This corresponds to the upper range of flow rates tested in Ref. [14]. The $1 \mathrm{~mm}$ and $0.5 \mathrm{~mm}$ resolutions were computed on in-house on a cluster with Intel ${ }^{\circledR} \mathrm{Xeon}^{\circledR}$ CPU E5-2630 v3 @ 2.40GHz processors and using 60 cores each, requiring approximate wall-clock time of $2.5 \mathrm{hr}, 14.1 \mathrm{hr}$, respectively for a simulation time of $100 \mathrm{~ms}$. The finest mesh resolution was simulated with on a Cray XC30 cluster with Intel ${ }^{\circledR}$ Xeon ${ }^{\circledR}$ CPU E5-2670 @ 2.60GHz processors using 600 cores and required $25 \mathrm{hr}$ for $100 \mathrm{~ms}$ of simulation time.

A series of iso-surfaces of the liquid volume fraction at a value of $\alpha=0.5$ are shown in Figure 5 for a range of times starting from $6 \mathrm{~ms}$ to $20 \mathrm{~ms}$ for the $\Delta x=0.25 \mathrm{~mm}$. After $6 \mathrm{~ms}$ the liquid jet has emerged from the nozzle but not yet impinged on the disk. After $7 \mathrm{~ms}$, the jet impinges on the disk and forms a high velocity liquid film on the surface of the disk as pressure forces the flow outwards. At $8 \mathrm{~ms}$ the liquid film leaves the surface of the disk and forms a liquid sheet. At the leading edge of the sheet ligaments and droplets are formed and subsequently shed due to the high velocity film entering a stagnant gas-phase flow. The liquid sheet continues to spread outwards, shedding droplets along the way. By $13 \mathrm{~ms}$, the high velocities induced by the initial jet impingement (and observed at $9 \mathrm{~ms}$ ) have resided. By $20 \mathrm{~ms}$ the liquid sheet has reached a radial distance of $\sim 70 \mathrm{~mm}$ from the center of the disk.

As the sheet transports radially outward, the sheet velocity decreases due to momentum exchange with the surrounding air. Eventually, due to the sheet thinning as it expands radially and due to instabilities in the flow, the sheet breaks up. Experimental observation suggests that for this flow rate and disk/nozzle configuration, the sheet transitions into ligaments and droplets at a radius of $\sim 165 \mathrm{~mm}$ from the disk center [14]. In the simulations, the sheet breakup occurs at a radius of about $25 \mathrm{~mm}, 160 \mathrm{~mm}$, and $155 \mathrm{~mm}$ for the three mesh resolution results shown in Figure 6. The $1 \mathrm{~mm}$ mesh resolution case clearly under predicts the sheet breakup distance, due to a lack of refinement necessary to sufficiently resolve the phase interface. Refining the mesh to $0.5 \mathrm{~mm}$ and subsequently to $0.25 \mathrm{~mm}$ results in a breakup distance that closely matches the experimental observation to within $10 \mathrm{~mm}$. For these cases, the sheet falls below the local refinement zone at a radial distance between 150 and $200 \mathrm{~mm}$. 

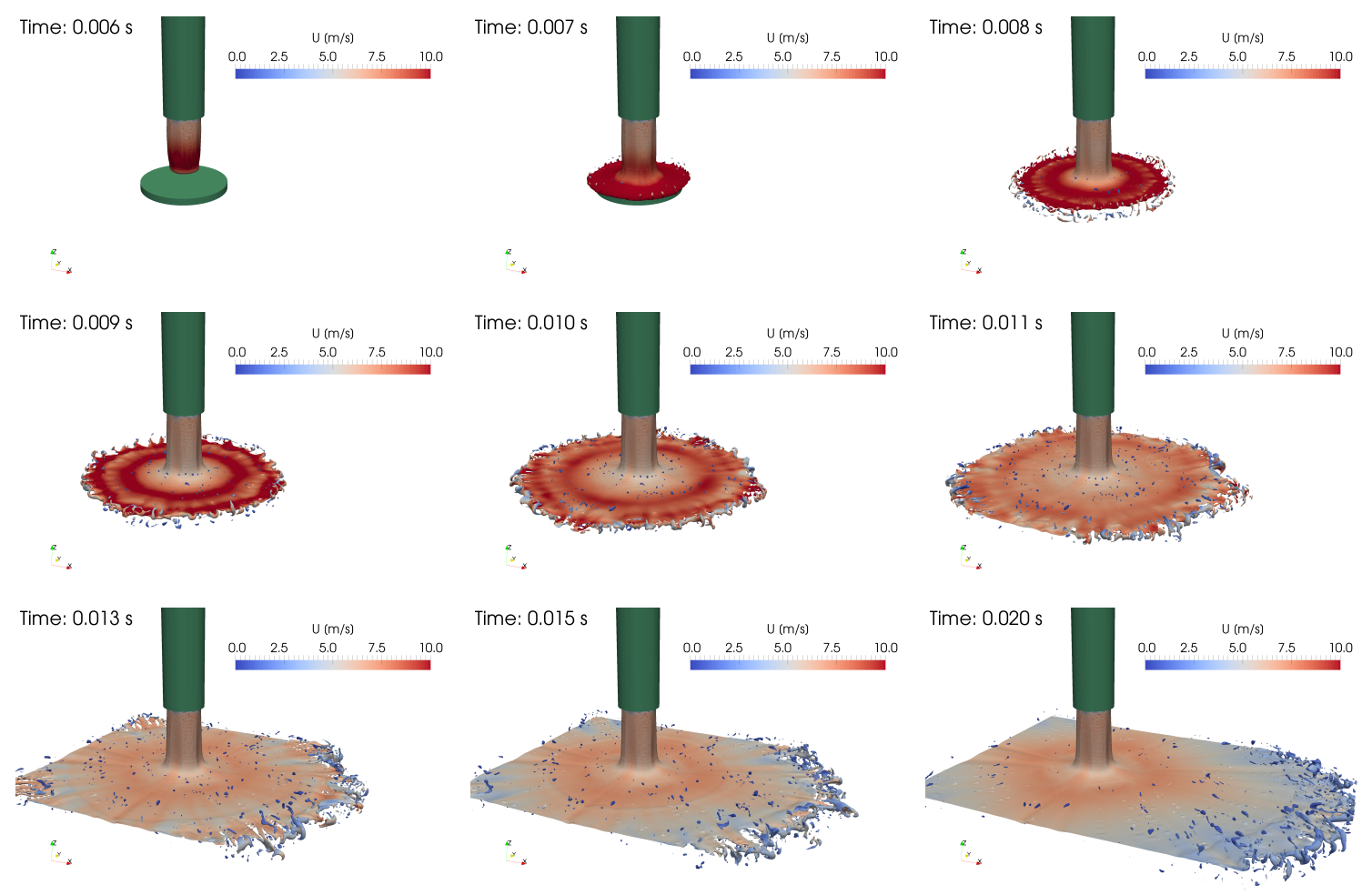

Figure 5. Iso-contours for liquid phase fraction $\alpha=0.5$ showing the phase interface at a series of times ranging from $6 \mathrm{~ms}$ to 20 $\mathrm{ms}$ for the $\Delta x=0.25 \mathrm{~mm}$ resolution case. Iso-contours are colored by velocity magnitude.

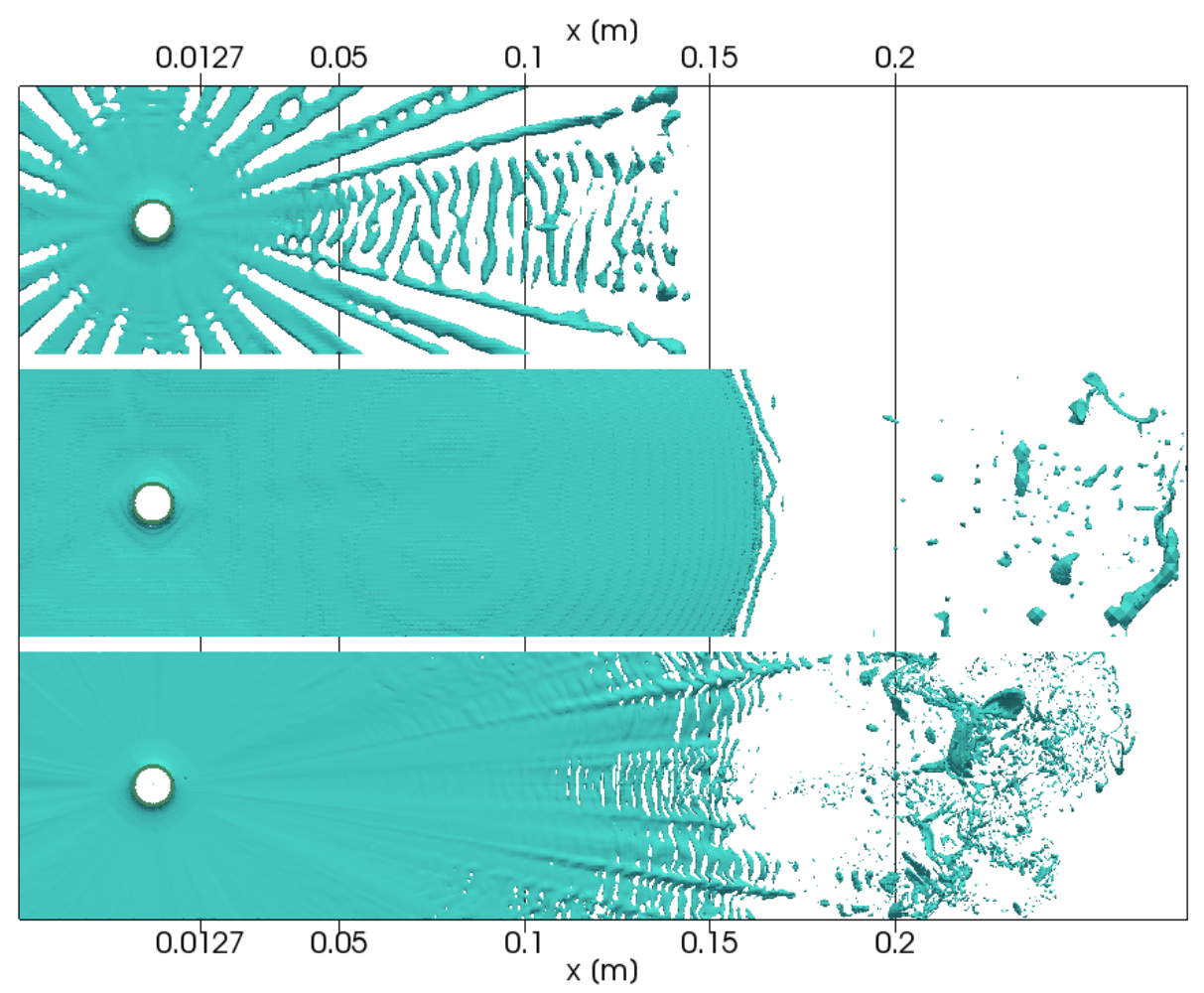

Figure 6. View in the $-\boldsymbol{z}$ direction of liquid surface iso-contours ( $\alpha=0.5)$ demonstrating the sheet breakup distances for the three mesh resolutions: a) $\Delta x=1 \mathrm{~mm}$, b) $\Delta x=0.5 \mathrm{~mm}$, and c) $\Delta x=0.25 \mathrm{~mm}$. 

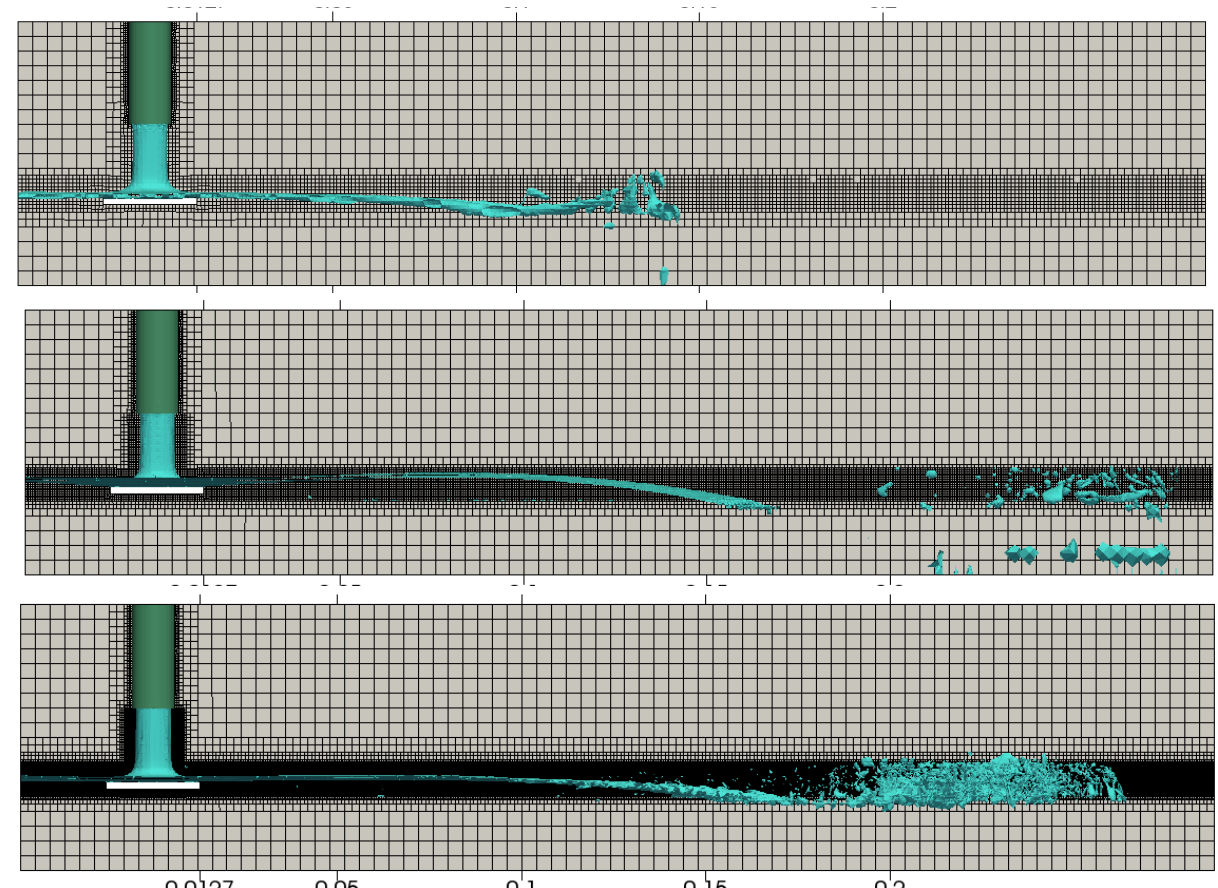

$0.0127 \quad 0.05$

0.1

$x(m)$

Figure 7. View in the $-y$ direction of the liquid surface $(\alpha=0.5)$ demonstrating the extent of the sheet breakup distance in relation to the mesh refinement regions.

A representation of the film flow on the surface of the disk is shown in Figure 8. The thickness of the liquid film as it exits the disk is of interest, as this sheet thickness influences the size distribution of droplets formed upon breakup. Experimentally, while direct measurements of the sheet thickness for this flow rate are not available, estimates for the film thickness at the edge of the disk based on analytical models place the value at $\sim 1.0$ to $1.2 \mathrm{~mm}$ [14]. The film thickness from the simulations, determined as the distance above the disk at which the liquid phase fraction drops to 0.5 , is shown in Table 2 . The simulated values are approximately in the expected range.

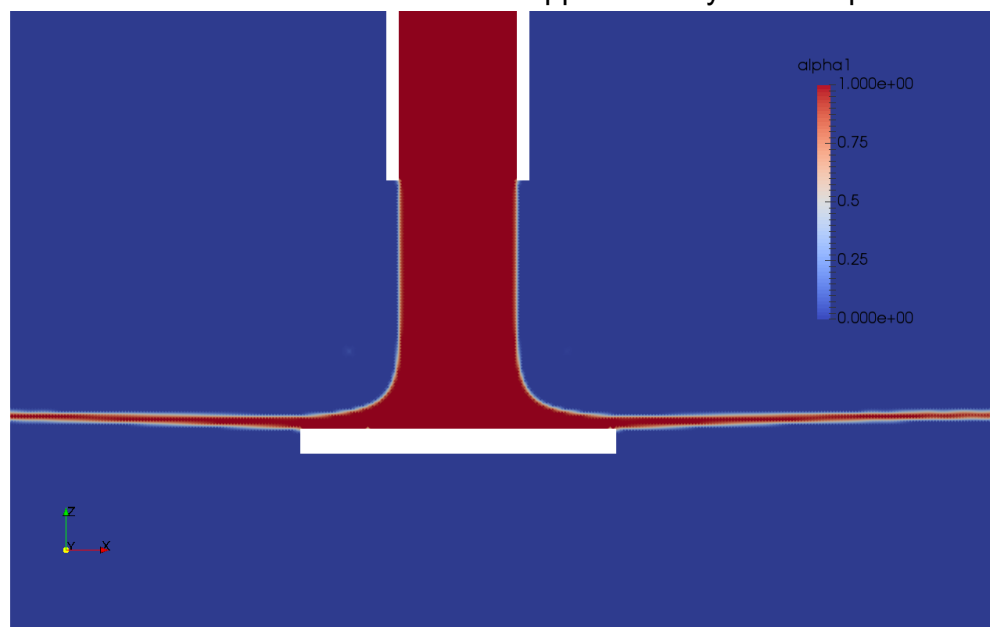

Figure 8. Representation of the liquid volume fraction in the near field of the nozzle and deflector for $\Delta x=0.25 \mathrm{~mm}$.

Table 2. Film thickness sampled at the edge of the disk for three mesh refinements.

\begin{tabular}{l|l|l|l|}
\multicolumn{2}{c}{$\Delta x=\mathbf{1 ~ m m}$} & \multicolumn{1}{c}{$\Delta x=\mathbf{0 . 5} \mathbf{m m}$} & $\mathbf{0 . 2 5} \mathbf{m m}$ \\
\hline Film thickness $[\mathrm{mm}]$ & 1.33 & 1.29 & 1.03
\end{tabular}

\section{Summary and Conclusions}

Simulating the atomization processes of a sprinkler has the potential to allow for enhanced insight into the key physics and controlling parameters. This approach also has the potential to be used as a predictive tool to estimate 
the spray injection profiles. In this study, the atomization of an idealized sprinkler geometry was simulated and comparisons were made to experimental data. An innovative isoAdvector scheme allowed for maintaining a sharp interface even with relatively coarse mesh resolution. A mesh refinement study was used to identify requirements for simulating sheet breakup distance. Mesh resolutions of $1 \mathrm{~mm}$ provided insufficient refinement, as the sheet breakup distance was greatly under predicted due to numerical diffusion of the interface, while a mesh resolution of $0.5 \mathrm{~mm}$ closely matched the experimentally determined value. A final mesh resolution of $0.25 \mathrm{~mm}$ was also simulated, resulting in finer detail in the region of the flow following sheet breakup. Ultimately, a mesh resolution of less than $0.25 \mathrm{~mm}$ will be required to resolve the flow features necessary to accurately predict atomization processes. Comparisons for film thickness predictions were also made, showing the simulated values for the finest mesh resolution closely resemble the values estimated from analytical models.

This study establishes the feasibility of using VOF modeling to adequately obtain injection patterns. The numerical model can subsequently be used to provide insight into sprinkler geometry effects on spray characteristics and to eventually lead to simulating realistic sprinkler geometries to obtain the atomization results necessary to initialize the sprinkler spray in fire suppression simulations.

\section{Acknowledgements}

This work was funded by FM Global as part of the Strategic Research Program for Fire and Suppression Modeling. Drs. Hrv Jasak and Vuko Vukčević of Wikki Ltd. are gratefully acknowledged for their guidance in using the isoAdvector and ghost-fluid-method schemes. For part of the simulations, this research used resources of the Oak Ridge Leadership Computing Facility at the Oak Ridge National Laboratory, which is supported by the Office of Science of the U.S. Department of Energy under Contract No. DE-AC05-00OR22725.

\section{References}

[1] Ren, N., Baum, H. R., and Marshall, A. W., 2011, "A comprehensive methodology for characterizing sprinkler sprays," Proceedings of the Combustion Institute, 33(2), pp. 2547-2554.

[2] Yu, H. Z., 1986, "Investigation of Spray Patterns of Selected Sprinklers with the FMRC Drop Size Measuring System," Proceedings of the 1st International Symposium on Fire Safety Science, pp. 1165-1176.

[3] Chan, T. S., Kung, H. C., Yu, H. Z., and Brown, W. R., 1994, "Experimental Study of Actual Delivered Density for Rack-Storage Fires," Proc. of the 4th International Symposium on Fire Safety Science, pp. 913-924.

[4] Widmann, J. F., 2001, "Phase Doppler Interferometry Measurements in Water Spray Produced by Residential Fire Sprinklers," Fire Safety Journal, 36, pp. 545-567.

[5] Sheppard, D. T., 2002, "Spray Characteristics of Fire Sprinklers," National Institute of Standards and Technology.

[6] Zhou, X., D'Aniello, S., and Yu, H. Z., 2010, "Spray Pattern Measurements of Selected Fire Sprinklers," Proceedings of the 12th Fire Science and Engineering Conference, INTERFLAM, Nottingham, UK, pp. 177-188.

[7] Zhou, X., D'Aniello, S. P., and Yu, H. Z., 2012, "Spray characterization measurements of a pendent fire sprinkler," Fire Safety Journal, 54(0), pp. 36-48.

[8] Wang, Y., Meredith, K. V., Zhou, X., Chatterjee, P., Xin, Y., Chaos, M., Ren, N., and Dorofeev, S. B., 2013, "Numerical Simulation of Sprinkler Suppression of Rack Storage Fires," Fire Safety Science.

[9] Hirt, C. W., and Nichols, B. D., 1981, "Volume of fluid (VOF) method for the dynamics of free boundaries," Journal of Computational Physics, 39(1), pp. 201-225.

[10] Jasak, H., and Weller, H. G., 1995, "Interface Tracking Capabilities of the Inter-Gamma Differencing Scheme," Technical Report, Imperial College.

[11] Roenby, J., Bredmose, H., and Jasak, H., 2016, "A computational method for sharp interface advection," Royal Society Publishing open sci, 3(160405).

[12] Vukčević, V., Jasak, H., and Malenica, S. i., 2016, "Decomposition model for naval hydrodynamic applications, Part I: Computational method," Ocean Engineering, 121, pp. 37-46.

[13] Vukčević, V., Jasak, H., and Malenica, S. i., 2016, "Decomposition model for naval hydrodynamic applications, Part II: Verification and validation," Ocean Engineering, 121, pp. 76-88.

[14] Zhou, X., and Yu, H.-Z., 2011, "Experimental investigation of spray formation as affected by sprinkler geometry," Fire Safety Journal, 46, pp. 140-150.

[15] 2016, "foam-extend," http://www.sourceforge.net/projects/foam-extend.

[16] Weller, H. G., Tabor, G., Jasak, H., and Fureby, C., 1998, "A tensorial approach to computational continuum mechanics using object-oriented techniques," Computers in Physics, 12(6), pp. 620-631.

[17] Brackbill, J. U., Kothe, D. B., and Zemach, C., 1992, "A continuum method for modeling surface tension," Journal of Computational Physics, 100(2), pp. 335-354.

[18] Suraj, S. D., Lakshman, A., and Mario, F. T., 2012, "Evaluating the performance of the two-phase flow solver interFoam," Computational Science \& Discovery, 5(1), p. 014016.

[19] Spalart, P. R., and Allmaras, S. R., 1992, "A One-Equation Turbulence Model for Aerodynamic Flows," AIAA Paper 92-0439. 